\title{
Stereotactic ablative body radiotherapy for ventricular tachycardia: An alternative therapy for refractory patients
}

\author{
Carolina De la Pinta* (iD, Raquel Besse** (D) \\ Departments of *Radiation Oncology, and **Internal Medicine, Ramón y Cajal Hospital; Madrid-Spain
}

\section{ABSTRACT}

Stereotactic ablative body radiotherapy (SABR) allows the administration of ablative doses of radiation in a focused form with a low probability of side effects and is widely used for cancer treatment. However, in the recent years its usefulness in benign cardiac pathology is being studied. In this study, we aimed to guide the cardiologist in SABR and its applications in treatment of refractory ventricular tachycardia. In this review, we analyzed published literature on PubMed and MEDLINE with papers published in the past 5 years. We included papers in the English language with information about indications, radiotherapy plan, doses and fractionations, and outcomes. All citations were evaluated for relevant content and validation.

Keywords: stereotactic ablative body radiotherapy, radiosurgery, ventricular tachycardia, radiotherapy

Cite this article as: De la Pinta $C$, Besse $R$. Stereotactic ablative body radiotherapy for ventricular tachycardia: An alternative therapy for refractory patients. Anatol J Cardiol 2021; 25: 858-62.

\section{Introduction}

Ventricular tachycardia (VT) is a potentially fatal type of arrhythmia because it can cause ventricular fibrillation or death. The causes can be very varied, sometimes occurring as a result of structural heart disease, such as acute myocardial infarction, or heart failure. At other times, it appears without any other apparent heart disease or as a consequence of certain medications. The most common symptoms are palpitations, dizziness, dyspnea, chest pain, or general discomfort.

In patients with structural heart disease, the myocardial scar after a myocardial infarction or fibrosis replacement is a common substrate of monomorphic ventricular tachycardia in a delayed manner. The extent of the myocardial necrosis and the degree of left ventricular dysfunction are important factors in determining the risk of arrhythmia after an infarction. Monomorphic VT usually appears in patients with a more extensive infarction and who also have a limited left ventricular ejection fraction. Classically, the incidence of post-infarction VT is $3 \%-5 \%$. The risk of VT in the general population has remained stable. However, it might be increasing as a result of improved post-infarction survival and the possibility of VT appearing years after the initial infarction combined with the progressive aging of the population (1). There are emerging data that border zones of therapy lead to the development of substrate for new VTs that have been targeted with ablation. 


\section{HIGHLIGHTS}

- In this study, we aimed to review a new treatment modality for ventricular tachycardia (VT) in patients refractory to medical treatment and radiofrequency.

- Stereotactic ablative body radiotherapy allows the administration of ablative radiation doses on the previously defined arrhythmogenic focus.

- We demonstrated the treatment workflow and results in these patients.

- This review guides the implementation of the treatment modality in a radiotherapy oncology department with the essential collaboration of cardiology specialists.

Pharmacological treatment of VTs includes antiarrhythmics. However, antiarrhythmics have a limited effect and extensive side effects. Invasive treatments include radiofrequency ablation, which produces tissue necrosis; alcohol ablation; or cryoablation. In alcohol ablation, ethanol is injected into the coronary artery branch, producing a surrounding myocardial infarction focus. However, the major complication is the inability to control the extent of the infarct tissue and the difficulty in producing the infarction at the focus. Recurrence is frequent, between $30 \%-60 \%$ of cases, recovering the lost electrical conduction. Recurrences are explained by multiple arrhythmogenic circuits or in-depth circuits that cannot be treated with conventional therapies (2). Catheter ablation is complicated when it is inaccessible owing to deep locations or when access is limited by metallic cardiac valves or thrombus. For these locations, other treatment modalities have been developed in the recent years (3). In addition, a special needle is available for radiofrequency at depth, although there is a risk of perforation or cardiac tamponade. Therefore, a non-invasive technique is considered necessary for these patients. The implantable cardioverter defibrillator (ICD) is highly effective in preventing death in these patients; however, the shocks are painful and impact the quality of life of these patients. This situation is associated with increased mortality and progression of heart failure.

Stereotactic ablative radiotherapy (SABR) or stereotactic body radiotherapy (SBRT) is a radiation therapy technique that allows high doses of radiation to be administered in a limited number of sessions (1-5). SABR is indicated in structural heart disease in patients with refractory to antiarrhythmic dose escalation and unsatisfactory ablation, monomorphic VT, or compassionate use among others. Contraindications to treatment include ICD dysfunction or any contraindication to radiosurgery (4). For administration, it is essential to identify the area producing VT with invasive or non-invasive mapping techniques, such as electrocardiography incorporating surface electrodes, and cardiac imaging. A Delphi consensus is to be published.

The mechanism is still unknown, but studies hypothesize that radiation therapy can produce vascular damage with hypoxia or necrosis and apoptotic cell death, resulting in fibrosis and scar formation weeks to months after the injury formation process. However, in the study of Jumeau et al. (5), the reduction in one patient was immediate, which may be associated with a phenomenon other than fibrosis. In animal models, inflammatory phenomena are thought to exist (6-8). In addition, late myocardial fibrosis and electrically inert tissue have been observed without evidence of acute or subacute damage after 6 months of follow-up.

In this review, our aim was to guide cardiologists in SABR and its applications in VT treatment. We analyzed published literature on PubMed and MEDLINE with papers published over the past 5 years. We included papers in the English language with information about SABR in VT. All citations were evaluated for relevant content and validation.

\section{Radiotherapy planning}

Dosimetric planning in SABR is performed on computed tomography (CT) images. The planning CT with contrast is performed in the supine position, and slides are made every $1 \mathrm{~mm}$ in most studies (9). We can use CT without contrast similar to the Radiosurgery for the Treatment of Refractory Ventricular Extrasystoles and Tachycardias (RAVENTA) protocol (4).

For patient immobilization, Lloyd et al. (10) and Cuculich et al. (11) used a vacuum bag. Cuculich et al. (11) and Robinson et al. (12) used abdominal compression too. The main problem in this treatment is heart mobility, which despite being somewhat reduced in patients with VT, the left atrium of these patients shows small translational movements, but significant upperinferior movement because of breathing. To control this, a fiducial marker can be used near the target, a temporary pacemaker, a stent, metallic cardiac valves, valvular or coronary calcifications, spinal bodies or ribs, or even the diaphragm as a surrogate marker of respiration. Cuculich et al. (11) used a CT in 4 dimensions acquiring a CT in 10 respiratory phases in free breathing and generating a contouring internal target volume. The patients were treated with cone beam CT without the need for fiducials. Neuwirth et al. (9), after recording images with 2 electrocardiograms, calculated the ITV considering heart contractions. To compensate for respiratory movements, the ICD was used as a fiducial marker for tracking.

It is very important to accurately detect the arrhythmogenic focus using single-photon emission CT (SPECT) imaging, magnetic resonance imaging (MRI), echocardiography and CT angiography (13). Cuculich et al. (11) used SPECT, MRI, and electrophysiological mapping. In the phase I/II study, ENCORE VT they used SPECT, MRI, ECG, and surface mapping for definition of the arrhythmogenic focus (14). Despite these studies, some authors argue that the exclusive use of the electrocardiogram could be sufficient without the need for further imaging tests. In the RAVENTA study (4), they used a multiphase electrocardiogram and an electrophysiological map with data from the right and left 
ventricles. In the study by Neuwirth et al. (9), electroanatomical 3D mapping was used to identify the re-entry circuit. After carrying out the tests, the electrophysiologist delimits the VT area according to the data from the electrophysiological register.

The planning target volume (PTV) is the margin generated by the technical uncertainty (14). The PTV varies from $0-5 \mathrm{~mm}$ according to studies. In the study by Neuwirth et al. (9), no margin was given to PTV, therefore, reducing the possibility of side effects. The organs at risk include the lungs, esophagus, stomach, and coronary arteries. The maximum dose (0.03 cc) was limited to $12 \mathrm{~Gy}$ in the coronary arteries (Fig. 1 describes inclusion criteria and radiotherapy process).

\section{Doses and fractionation}

Doses between $25 \mathrm{~Gy}$ and $35 \mathrm{~Gy}$ in single doses would be equivalent to radiofrequency ablation. Based on the literature, the most commonly used dose is $25 \mathrm{~Gy}$ in a single dose $(9,11)$, which is considered safe in preclinical studies (15). Jumeau et al. (5) used $25 \mathrm{~Gy}$ in 1 fraction, administering the treatment in 45 min with a conformality index of 1.24 , the median dose to the heart was $6 \mathrm{~Gy}$. The maximum dose $(<0.03 \mathrm{cc})$ to the coronary arteries was $12 \mathrm{~Gy}$ and $11.9 \mathrm{~Gy}$, respectively. Patients had no episodes of VT during treatment, and were extubated 3 days after the procedure. In the study by Blanck et al. (4) and Lloyd et al. (10), 95\% of the PTV received a dose of $25 \mathrm{~Gy}$ with a range of $110 \%-140 \%$. In the study by Neuwirth et al. (9), a PTV dose of 25 $\mathrm{Gy} / 1$ fraction was prescribed at an isodose of $80 \%(66 \%-84 \%)$. The Monte Carlo algorithm was used because of the proximity of the lungs. At present, there are insufficient data to increase or decrease the dose, although it is suggested that lower doses could be used. Administration of the treatment in less than 20 minutes is desirable in radiobiological terms (16). On the basis of the available studies, we believe that the most appropriate dose is $25 \mathrm{~Gy}$ in 1 fraction, although more studies and longer follow-up periods are needed.

\section{Outcomes and adverse events}

In October 2012, the first patient was treated (8). A 71-yearold male who presented a reduction in VT episodes from 562 to 52 in 2 months after SABR. However, it was not until the publication of Cuculich et al. (11) that this treatment option began to take hold. The patients included in this series presented with at least 3 episodes of VT in 3 months despite 2 anti-arrhythmogenic drugs and 2 catheter ablation at least, and they were not candi-

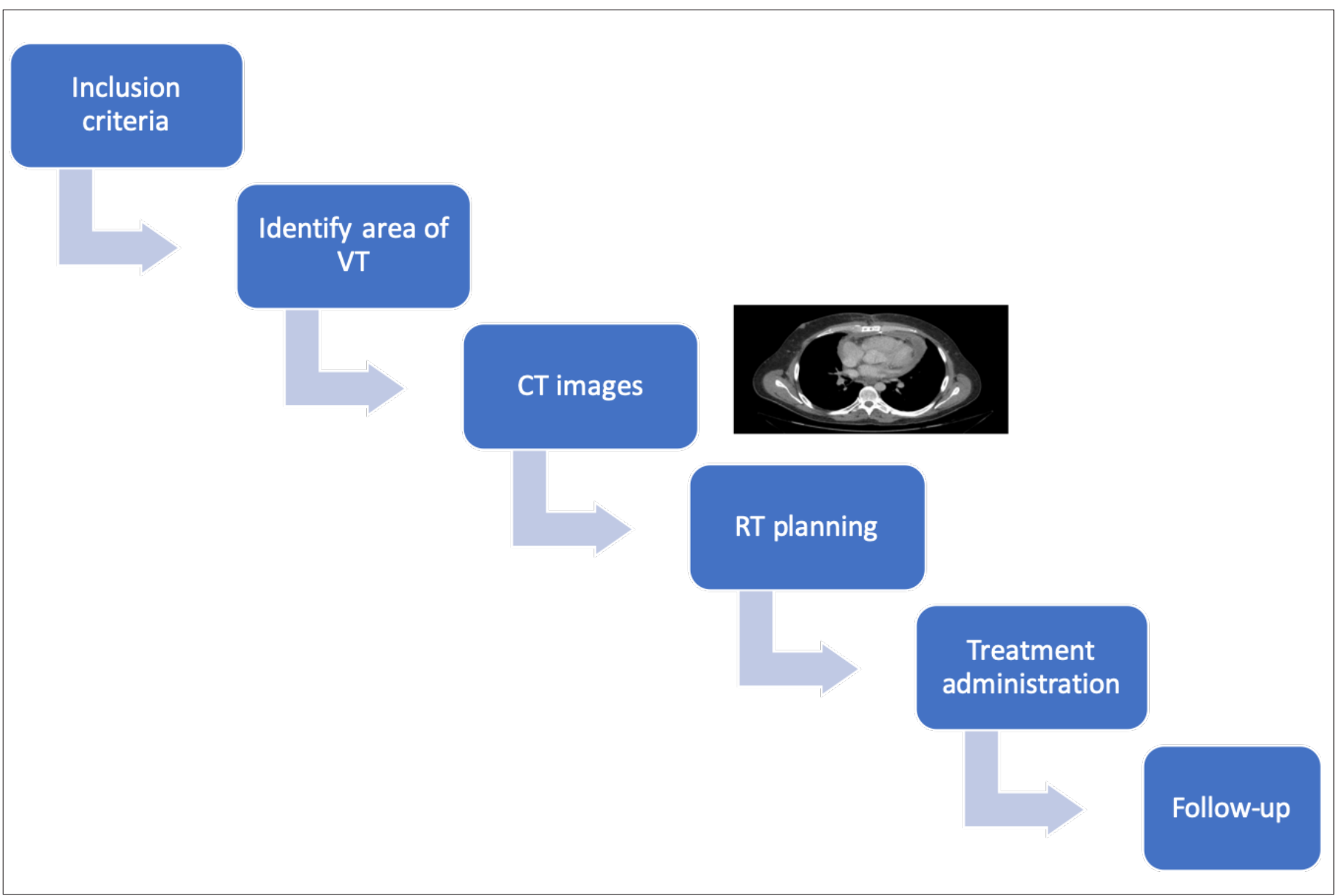

Figure 1. Inclusion criteria and radiotherapy process 
dates for a heart transplant. Cuculich et al. in 2017 (11) treated 5 patients with a mean age of 66 years (60-83 years) with doses of $25 \mathrm{~Gy} / 1 \mathrm{fr}$. Three months before treatment, the patients presented with 6,577 episodes at 6 weeks post SABR, 680 episodes, after those 6 weeks 4 episodes, with a baseline reduction of $99.9 \%$. The treatment can be administered regardless of depth, always using appropriate monitoring during treatment. The medium left ventricular ejection fraction was maintained. During follow-up, amiodarone was discontinued, and sinus node function recovered in 3 patients. Jumeau et al. (5) observed a lasting reduction in VT episodes in 5 patients with a median follow-up of one year. Robinson et al. (17) in a phase I/II included 19 patients with ischemic and non-ischemic cardiomyopathy. After treatment with SABR, the median number of episodes was 119 to $3(p<0.01)$, and overall survival was $89 \%$ at 6 months and $72 \%$ at 12 months. In a study by John et al. 2018 (18), 5 patients with adequate response without side effects were included. Lloyd et al. (10) included 10 patients in 2019, VT was triggered by ischemic failure in $40 \%$ of patients and non-ischemic in $60 \%$ of patients. The reduction in total shocks was $68 \%(2.9$ vs. 0.9 shocks/month); if the only non-responsive patient was excluded, it was $94 \%(p=0.04)$. Inclusion criteria included failure of 2 antiarrhythmic drugs, radiofrequency failure, and failure of an adjuvant treatment. One patient who had to be resuscitated during treatment and 2 patients who had mild radiographic pneumonitis were targeted as side effects. Three patients received a heart transplant after SABR. Microscopic analysis of the explant showed edema and vacuolization with mild fibrosis. Neuwirth et al. (9) included 10 patients. After a median follow-up of 28 months (16-54 months), VT episodes were reduced to $87.5 \%$ $(p=0.012)$. The mean at the time of the first antitachycardia pacing and shock was 6.5 and 21 months, respectively. After the blancking period, VT recurred in 8 patients. Of the 8 patients, only 2 of them experienced recurrences of VT at 3-6 months of SABR. Amiodorone was restarted in 2 patients with limited efficacy. During follow-up, troponin levels did not rise significantly, and NT-pro-BNP levels did not increase $(\mathrm{p}=0.203$ and $\mathrm{p}=0.386)$. The results of the study by the Gianni et al. (19) confirmed an initial reduction after $25 \mathrm{~Gy} / 1 \mathrm{fr}$, but did not result in effective arrhythmia control in the long term.

Acute effects were reported in the first 30 days of treatment. The target period was 6 weeks. Side effects described in the literature include valve damage, early coronary disease, pericardial disease, intramyocardial damage, phrenic nerve damage, esophageal damage, and lung damage, among others. In the study by Cuculich et al. (11), there were no complications during treatment or during hospitalization. One patient died of a fatal stroke 3 weeks after treatment. Lung opacities consisting of moderate inflammatory changes were seen at 3 months and resolved within 1 year. Three patients had fatigue 1 day after treatment. There were no side effects on the ICD. In the study by Neuwirth et al. (9), 3 patients experienced ventricular storm, 4 had nausea, and 1 had progressive mitral regurgitation. Three patients died after 18,43 , and 54 months. The causes of death were vascular dementia with Alzheimer's disease in 1 patient, and heart failure in 2 others. There was no worsening of the left ventricular ejection fraction and no pericardial effusion. In the ENCORE VT (12) phase I/II study, 2 patients presented with grade 3 toxicity consisting of exacerbation of heart failure and pericarditis, 5 with asymptomatic pericardial effusion, and 2 with pneumonitis. In the study by Gianni et al. (19), patients did not have adverse effects in the first 90 days after treatment. These data are still very limited, and more studies are needed to evaluate the long-term effect of SBRT on myocardial tissue with some series describing scar tissue formation, which may be the new arrhythmogenic foci.

\section{Study limitations}

This study had several limitations including the characteristics of the studies and the limited number of patients. The methods of target delineation are heterogenous amongst centers with different technologies being used. Moreover, the patients who receive this treatment may be different depending on the inclusion criteria for treatment at different centers. There is a huge need for a prospective study evaluating this treatment technology.

SBRT in VT could become the therapeutic alternative after failure of other treatments, with a decrease in VT episodes and an improvement in quality of life. Optimal doses have not been determined. Further studies are needed to support its indication, optimal doses, and fractionation. Significant development of this treatment is expected in the coming years.

\section{Conclusion}

SABR is an option under study and development for medically and radiofrequency resistant VTs, with significant reduction in episodes in most of the studies analyzed and a low rate of side effects. Further studies are needed to establish this treatment as a conventional treatment for refractory VTs.

Conflict of interest: None declared.

Peer-review: Externally peer-reviewed.

Author contributions: Concept - C.D.P., R.B.; Design - C.D.P., R.B.; Supervision - C.D.P., R.B.; Fundings - C.D.P., R.B.; Materials - C.D.P., R.B.; Data collection \&/or processing - C.D.P., R.B.; Analysis \&/or interpretation - C.D.P., R.B.; Literature search - C.D.P., R.B.; Writing - C.D.P., R.B.; Critical review - C.D.P., R.B.

\section{References}

1. Benito B, Josephson ME. Ventricular tachycardia in coronary artery disease. Rev Esp Cardiol (Engl Ed) 2012; 65: 939-55. [Article in English, Spanish] [Crossref]

2. Yokokawa M, Desjardins B, Crawford T, Good E, Morady F, Bogun F. Reasons for recurrent ventricular tachycardia after catheter ablation of post-infarction ventricular tachycardia. J Am Coll Cardiol 2013; 61: 66-73. [Crossref] 
3. Kumar S, Barbhaiya CR, Sobieszczyk P, Eisenhauer AC, Couper GS, Nagashima K, et al. Role of alternative interventional procedures when endo- and epicardial catheter ablation attempts for ventricular arrhythmias fail. Circ Arrhythm Electrophysiol 2015; 8: 606-15. [Crossref]

4. Blanck O, Buergy D, Vens M, Eidinger L, Zaman A, Krug D, et al. Radiosurgery for ventricular tachycardia: preclinical and clinical evidence and study design for a German multi-center multi-platform feasibility trial (RAVENTA). Clin Res Cardiol 2020; 109: 1319-32. [Crossref]

5. Jumeau R, Ozsahin M, Schwitter J, Vallet V, Duclos F, Zeverino M, et al. Rescue procedure for an electrical storm using robotic noninvasive cardiac radio-ablation. Radiother Oncol 2018; 128: 189-91. [Crossref]

6. van der Ree MH, Blanck 0, Limpens J, Lee CH, Balgobind BV, Dieleman EMT, et al. Cardiac radioablation- A systematic review. Heart Rhythm 2020; 17: 1381-92. [Crossref]

7. Jumeau R, Ozsahin M, Schwitter J, Elicin O, Reichlin T, Roten L, et al. Stereotactic Radiotherapy for the Management of Refractory Ventricular Tachycardia: Promise and Future Directions. Front Cardiovasc Med 2020; 7: 108. [Crossref]

8. Loo BW Jr, Soltys SG, Wang L, Lo A, Fahimian BP, lagaru A, et al. Stereotactic ablative radiotherapy for the treatment of refractory cardiac ventricular arrhythmia. Circ Arrhythm Electrophysiol 2015; 8: 748-50. [Crossref]

9. Neuwirth R, Cvek J, Knybel L, Jiravsky 0, Molenda L, Kodaj M, et al. Stereotactic radiosurgery for ablation of ventricular tachycardia. Europace 2019; 21: 1088-95. [Crossref]

10. Lloyd MS, Wight J, Schneider F, Hoskins M, Attia T, Escott C, et al. Clinical experience of stereotactic body radiation for refractory ventricular tachycardia in advanced heart failure patients. Heart Rhythm 2020; 17: 415-22. [Crossref]

11. Cuculich PS, Schill MR, Kashani R, Mutic S, Lang A, Cooper D, et al. Noninvasive Cardiac Radiation for Ablation of Ventricular Tachycardia. N Engl J Med 2017; 377: 2325-36. [Crossref]
12. Robinson CG, Samson P, Moore KMS, Hugo GD, Knutson N, Mutic S. et al. Longer term results from a phase I/II Study of EP-guided Noninvasive Cardiac Radioabla $a$ tion for Treatment of Ventricular Tachycardia (ENCORE-VT). Int J Radiat Oncol Biol Phys 2019; 105: 682. [Crossref]

13. Zei PC, Wong D, Gardner E, Fogarty T, Maguire P. Safety and efficacy of stereotactic radioablation targeting pulmonary vein tissues in an experimen-tal model. Heart Rhythm 2018; 15: 1420-7. [Crossref]

14. Wang L, Fahimian B, Soltys S G, Zei P, Lo A, Gardner EA, et al. Stereotactic Arrhythmia Radioablation (STAR) of Ventricular Tachycardia: A Treatment Planning Study. Cureus 2016; 8: e694. [Crossref]

15. Lehmann HI, Deisher AJ, Takami M, Kruse JJ, Song L, Anderson $\mathrm{SE}$, et al. External Arrhythmia Ablation Using Photon Beams: Ablation of the Atrioventricular Junction in an Intact Animal Model. Circ Arrhythm Electrophysiol 2017; 10: e004304. [Crossref]

16. Schmitt D, Blanck O, Gauer T, Fix MK, Brunner TB, Fleckenstein J, et al. Technological quality requirements for stereotactic radiotherapy: Expert review group consensus from the DGMP Working Group for Physics and Technology in Stereotactic Radiotherapy. Strahlenther Onkol 2020; 196: 421-43. [Crossref]

17. Robinson CG, Samson PP, Moore KMS, Hugo GD, Knutson N, Mutic $\mathrm{S}$, et al. Phase I/II trial of Electrophysiology-Guided Non-invasive Cardiac Radioablation for Ventricular Tachycardia. Circulation 2019; 139: 313-21. [Crossref]

18. John RM, Shinohara ET, Price M, Stevenson WG. Radiotherapy for ablation of ventricular tachycardia: assessing collateral dosing. Comput Biol Med 2018; 102: 376-80. [Crossref]

19. Gianni C, Rivera D, Burkhardt JD, Pollard B, Gardner E, Maguire P, et al. Stereotactic arrhythmia radioablation for refractory scarrelated ventricular tachycardia. Heart Rhythm 2020; 17: 1241-8. [Crossref] 